\title{
Generation and control of extreme blueshifted continuum peaks in optical Kerr media
}

\author{
D. Faccio, ${ }^{1,5}$ A. Averchi, ${ }^{1,5}$ A. Lotti, ${ }^{1,5}$ M. Kolesik, ${ }^{2}$ J. V. Moloney, ${ }^{2}$ A. Couairon, ${ }^{3,5}$ and P. Di Trapani ${ }^{1,4,5}$ \\ ${ }^{1}$ CNISM and Department of Physics and Mathematics, Università dell'Insubria, Via Valleggio 11, IT-22100 Como, Italy \\ ${ }^{2}$ Arizona Center for Mathematical Sciences and Optical Sciences Center, University of Arizona, Tucson, Arizona 85721, USA \\ ${ }^{3}$ Centre de Physique Théorique, CNRS, École Polytechnique, F-91128, Palaiseau, France \\ ${ }^{4}$ Department of Quantum Electronics, Vilnius University, Sauletekio Avenue 9, bldg.3, LT-10222, Vilnius, Lithuania \\ ${ }^{5}$ Virtual Institute for Nonlinear Optics, Centro di Cultura Scientifica Alessandro Volta, \\ Villa Olmo, Via Simone Cantoni 1, 22100 Como, Italy \\ (Received 19 June 2008; published 18 September 2008)
}

\begin{abstract}
We demonstrate tunable, extremely blueshifted continuum in $\lambda=1.055 \mu \mathrm{m}$ ultrashort laser pulse filamentation in silica. Close to threshold, the continuum appears as a single, isolated blue peak. The spectral position of the two supercontinuum components can be tuned and a regime with encompassing fundamental and second harmonic is possible to achieve. At higher energies, the continuum expands in bandwidth starting from the blue peak. The spectral dynamics and tunability are explained in terms of $X$-wave generation and intrafilament pulse splitting which may be controlled by modifying the input pulse focusing conditions.
\end{abstract}

DOI: 10.1103/PhysRevA.78.033825

PACS number(s): 42.65.Re, 42.65.Ky, 42.65.Jx

\section{INTRODUCTION}

Supercontinuum (SC) generation, i.e., the generation of an ultrabroadband spectrum starting from a narrowbandwidth laser pulse, is attracting much interest due to the potential applications in a wide range of areas such as LIDAR [1], few-cycle pulse generation [2], and ultraviolet spectroscopy [3]. Although in general the physical processes lying at the heart of SC generation are the same in all Kerr media and are well known, e.g., self-phase modulation (SPM), four-wave mixing (FWM), shock-front formation, etc. [3], there are still a number of features, such as the formation of spectrally isolated blueshifted peaks described below, whose true nature remains rather elusive. In bulk media the input pulse collapses assuming a self-similar Townes profile $[4,5]$. As the intensity increases the collapse is arrested by a saturating mechanism such as nonlinear losses or self-generated-plasma defocusing and a filament is formed, i.e., a tightly focused peak that maintains a very small diameter for many diffraction lengths [6]. Strong spatiotemporal instabilities are observed that give rise to SC generation and a new feature with respect to the fiber case, known as conical emission, i.e., the generation of angularly dispersed frequencies with the emission angle that increases with increasing frequency shift. Conical emission is now recognized as a direct manifestation of space-time dynamics [7] and of the formation of $X$ waves $[9,8]$. $X$ waves are a particular example of conical waves and form a continuous family of stationary solutions to the wave propagation equation in both the linear [10] and nonlinear regime [11]. This family is parametrized by the phase and group velocities which may, in principle, take on any particular value. Filamentation has thus been interpreted as the spontaneous formation and interaction of $X$ waves [12]: pulse splitting is related to the formation of two $X$ waves with opposite group velocities (in the moving reference frame on the input pulse) while SC appears as a necessary step in the $X$-wave formation process [14].

Laser pulse filamentation in the anomalous groupvelocity-dispersion (GVD) region has been investigated, with particular attention to spectral reshaping, numerically in 2D planar waveguides highlighting similar dynamics to the $1 \mathrm{D}$ case [13], and both experimentally and numerically in 3D bulk media [15-17]. A localized pulse is formed together with a strongly blueshifted peak that exhibits a marked angular dispersion that in the 3D case finds a natural description in the so-called "Fish" conical wave [15]. More recently, attention has been given to a similar blueshifted peak observed with a pump pulse in the normal GVD regime and far away from the zero GVD wavelength [18]. A tunable blue peak in the continuum spectrum was generated by tuning the chirp of the input pump pulse, with a maximum shift of about $300 \mathrm{~nm}$. This blue peak was roughly $5 \mathrm{~dB}$ (a factor 3 ) more intense than the marked SC and thus spectrally "connected" to the pump pulse and has been explained in terms of self-steepening.

\section{EXPERIMENTS}

Here we study the formation of radiation that is strongly blueshifted over more than an octave. The blue peak is associated with a collapsing pump pulse in bulk fused silica in the normal GVD regime and is connected to the pump wavelength only by an extremely weak continuum (a factor $\sim 100$ times less intense) that was in some cases not even experimentally detectable. This blue spectral peak is shown to be the blueshifted tail of the same pump $X$ wave that is formed in the filament. In particular, the position of the blueshifted tail depends on the $X$-wave group velocity. The latter may be continuously tuned by controlling the input pulse parameters. Our numerics shed light onto the evolution of the blue peak in connection with the pump $X$ wave: the $X$ wave accelerates during propagation and in doing so it accordingly modifies the spectral location of the blue peak leading to an overall broadening of the SC.

Experiments were performed with a 1 ps duration [full width at half maximum (FWHM)], $1055 \mathrm{~nm}$ laser pulse delivered by a $10 \mathrm{~Hz}$, amplified Nd:glass laser (Twinkle, Light Conversion Ltd., Vilnius, Lithuania). The pulse had a diam- 


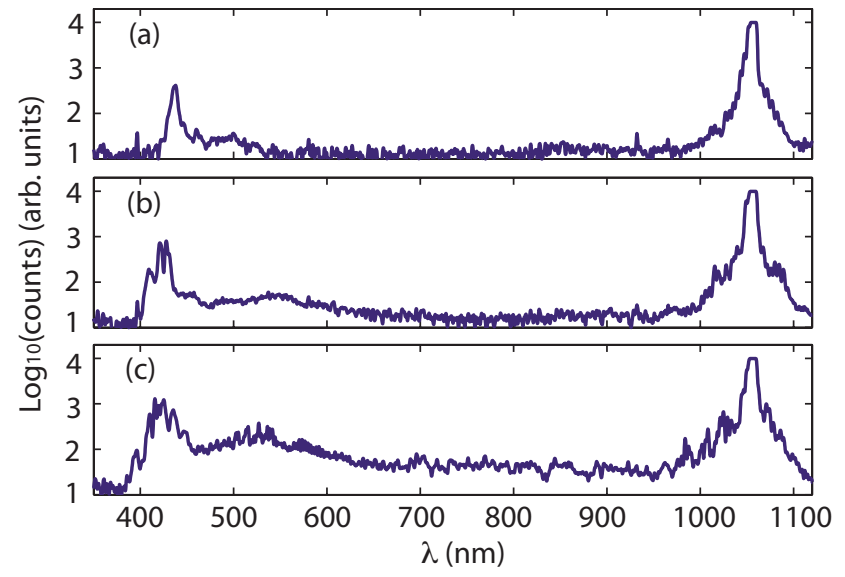

FIG. 1. (Color online) Experimental spectra generated from a 1-cm-long sample of fused silica placed at $52 \mathrm{~cm}$ from the focusing lens and with increasing input energy: (a) $20 \mu \mathrm{J}$, (b) $30 \mu \mathrm{J}$, and (c) $50 \mu \mathrm{J}$.

eter of $5 \mathrm{~mm}$ (FWHM) and was focused into a $2 \mathrm{~cm}$ long sample of fused silica using a $52 \mathrm{~cm}$ focal length lens. The input energy was adjusted using a first order half-wave plate and a polarizer. Figure 1 shows the spectra recorded using a 14-bit fiber-coupled spectrometer (Ocean Optics) when the sample is placed at $52 \mathrm{~cm}$ from the lens and with (a) $20 \mu \mathrm{J}$, (b) $30 \mu \mathrm{J}$, and (c) $50 \mu \mathrm{J}$ input energy. The pump pulse is saturated so as to highlight the weaker spectral features. As may be seen, at the threshold input energy of $20 \mu \mathrm{J}$ a single distinct, apparently spectrally isolated blue peak forms at $450 \mathrm{~nm}$, with more than an octave shift from the pump wavelength. The total energy in this peak was roughly $100 \mathrm{~nJ}$ corresponding to a conversion efficiency of $0.5 \%$. Increasing the input energy leads to the formation of a broadband emission between 400 and $700 \mathrm{~nm}$. The notable feature of this bandwidth increase is that rather than extending from the pump spectrum, as is commonly observed and as would be expected for a generation process related to the pump SPM or self-steepening, the SC grows starting from the $450 \mathrm{~nm}$ blue peak. We also note that, differently from Ref. [8], no redshifted peaks (with respect to the pump frequency) were observed in the spectrum.

In Figs. 2(a) and 2(b) we show the angularly resolved spectra, measured with a commercial imaging spectrometer (Lot-Oriel, MS260i) and recorded with a modified digital Nikon D70 camera, for the same settings as in Fig. 1 and for input energies of 20 and $40 \mu \mathrm{J}$, respectively. In Fig. 2(a) we note the onset of weak conical emission around the pump, indicating that we are at the threshold for filamentation. Most importantly, the angular dispersion of the blue peak also starts to be discernible close to threshold. We may fit the conical emission at the pump wavelength, $\lambda_{0}=2 \pi c / \omega_{0}$ using the $X$-wave relation $k_{\perp}=\sqrt{k^{2}-k_{z}^{2}}$ with $k=(\omega / c) n(\omega)$ and

$$
k_{z}(\omega)=k\left(\omega_{0}\right)+\frac{\omega-\omega_{0}}{v_{X}},
$$

where $v_{X}$ is the group velocity of the $X$-wave (i.e., the split pulse) Eq. (1) may be derived by simply requiring that the $X$

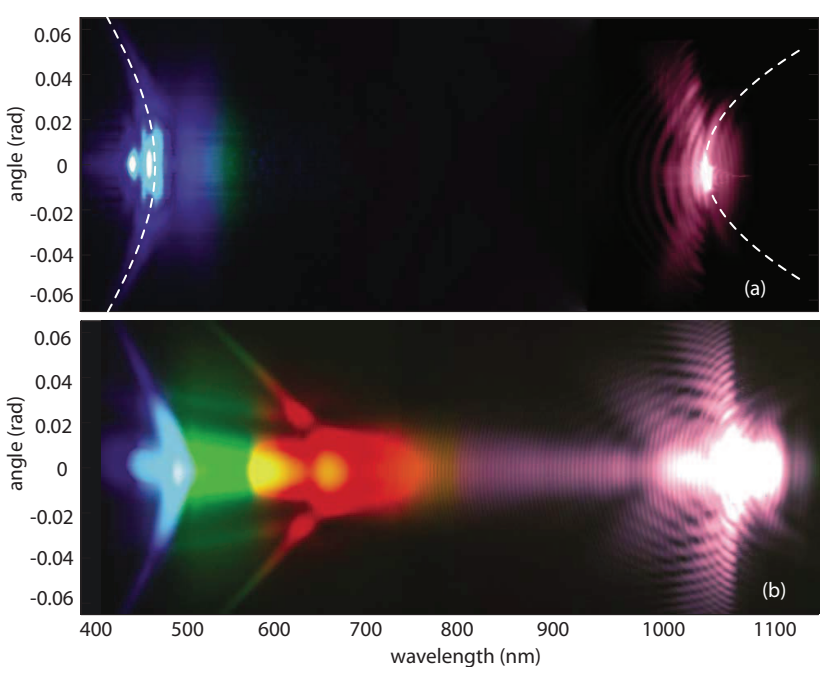

FIG. 2. (Color online) Experimental angularly resolved spectra for input pulse energies of (a) $20 \mu \mathrm{J}$ and (b) $40 \mu \mathrm{J}$. The dashed white lines show a plot of Eq. (1) with $v_{X}=2.035 \times 10^{8} \mathrm{~m} / \mathrm{s}$.

wave does not suffer dispersion, i.e., $d^{2} k_{z} / d \omega^{2}=0$. However, it may also derived from the effective three wave mixing (ETWM) model [17]: the Kerr-induced pulse reshaping is described in terms of a scattering process in which the input pump pulse generates a nonlinear polarization wave in the medium which in turn scatters the pump pulse. The phase matching relation for this process is given precisely by Eq. (1) in which $v_{X}$ is interpreted as the group velocity of the nonlinear polarization wave. We note that as $v_{X}$ is derived from the angular spectrum, Eq. (1) has no free parameters. The white dashed line in Fig. 2(a) shows the plot of Eq. (1) with $v_{X}=2.035 \times 10^{8} \mathrm{~m} / \mathrm{s}$. As may be seen the curve explains the conical emission around both the pump wavelength and the blue peak, while it has no solutions for frequencies in the interval between the pump and the blue peak. In particular, the position of the blue peak is determined by the precise value of $v_{X}$ : larger splitting velocities lead to larger wavelength gaps between the pump and the blueshifted $X$ tails. Following the same arguments as in Ref. [8], the good overlap of Eq. (1) with the measured spectral features indicates that, although in the experiments the blue peak appears to be spectrally isolated from the pump pulse, it should actually be considered in the near field as part of the same, single subluminal $X$ wave originating from the pump pulse. Figure 2(b) shows that at higher input energies the blue peak maintains its main characteristics. The spectrum is now broadened and particularly intense between 450 and $700 \mathrm{~nm}$. We note that the continuum does not extend significantly toward shorter wavelengths and remains contained between the pump and blue $X$ tails. We also underline the new spectral peak close to $650 \mathrm{~nm}$ that also exhibits a marked angular dispersion. We interpret this as due to the higher input energy that leads to a second pulse splitting process within the fully formed filament [12]. Indeed, it has been pointed out that the pulse splitting velocity, which is also the group velocity $v_{X}$ of the $X$ waves, depends on the pump pulse peak intensity at the moment of tightest nonlinear focus [8]. It is expected that separate pulse splitting cycles within the 


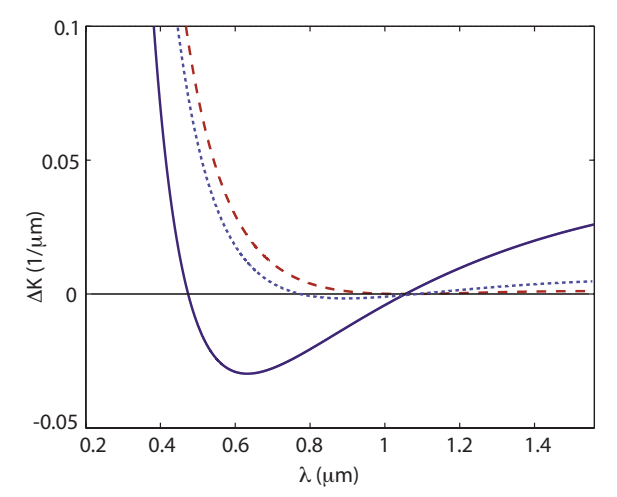

FIG. 3. (Color online) Longitudinal $\left(k_{\perp}=0\right)$ phase mismatch calculated from Eq. (1) for three different cases: $v_{X}=v_{g}$ $=(d k / d \omega)^{-1}=2.052 \times 10^{8} \mathrm{~m} / \mathrm{s} \quad($ red dashed line $), \quad v_{X}=2.048$ $\times 10^{8} \mathrm{~m} / \mathrm{s}$ (blue dotted line), and $v_{X}=2.035 \times 10^{8} \mathrm{~m} / \mathrm{s}$ (blue solid line). Only pulse splitting events in which the trailing peak velocity $v_{x}$ is sufficiently different from the group velocity, as in the third case shown, will generate a well separated blue peak in the supercontinuum spectrum.

filament will lead to distinct peak intensities. Therefore, the $X$ waves that emerge from distinct splitting events will manifest themselves in the spectrum as angularly dispersed emission peaks centered at distinct wavelengths.

In order to emphasize the role played by the group velocity of the filament pulse in generating the SC features, lets consider just the on-axis dynamics of the pulse. In Fig. 3 we plot the on-axis phase mismatch $\Delta K$ for the scattering process described by $\Delta K=k(\omega)-k_{z}(\omega)$, where $k_{z}(\omega)$ is given by Eq. (1) for three different values of $v_{X}$. If $v_{X}$ is determined solely by material dispersion $v_{X}=v_{g}=(d k / d \omega)^{-1}$, i.e., the pulse is propagating with the same group velocity of the input Gaussian pulse, then $\Delta K>0$ for all wavelengths and no instability in the form of a blueshifted peak is expected to appear (red dotted line). If $v_{X}$ is slightly smaller than the material group velocity then $\Delta K \sim 0$ for wavelengths between $1055 \mathrm{~nm}$ and $\sim 800 \mathrm{~nm}$ (blue dashed line). So the input pulse will be efficiently scattered from the material nonlinear polarization wave within this wavelength range and the SC will appear as originating from the pump spectrum. This is the typical situation observed in most filamentation regimes. Conversely, if the $v_{X}$ is significantly different from $v_{g}$ then $\Delta K$ is large everywhere except in the close vicinity of a strongly blueshifted wavelength (the blue solid line shows the $\Delta K$ curve for the value $v_{X}=2.035 \times 10^{8} \mathrm{~m} / \mathrm{s}$ determined from Fig. 2). Therefore, the SC will no longer develop starting from the pump and the phase-matched scattering process will appear as a blue peak, eventually with only extremely weak scattered light in the spectral region separating it from the pump, as observed in our experiments.

We now address the possibility to tune the position of the blue peak in the spectrum and therefore control the SC generation process. The simplest experimental option is to tune the input focusing condition. Figures 4(a)-4(f) show the spectra recorded with the sample placed at $z=49,50,50.5$, 51,52 , and $53 \mathrm{~cm}$ from the input focusing lens (which had a fixed $52 \mathrm{~cm}$ focal length). The energy for each measurement was adjusted in each case so as to be just slightly above the

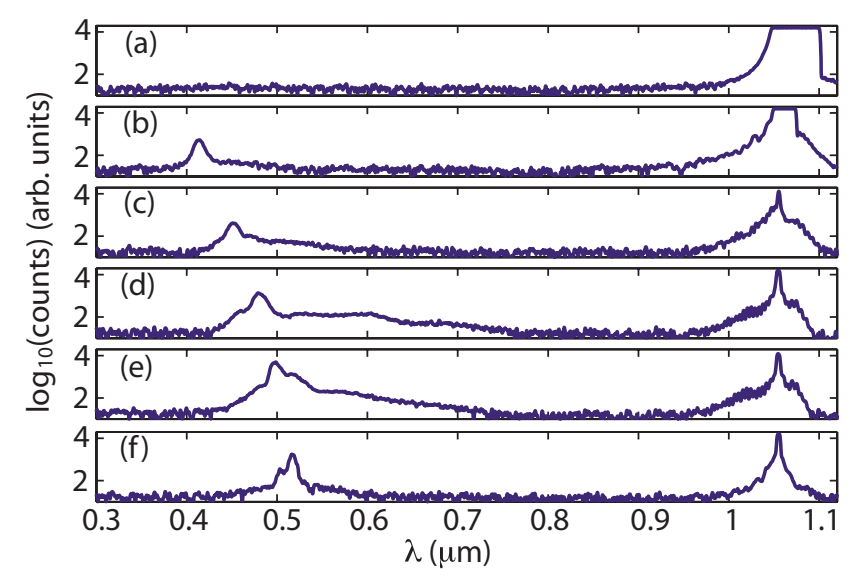

FIG. 4. (Color online) Spectra measured for different positions of the fused sample with respect to the input focusing lens. (a) $49 \mathrm{~cm}$, (b) $50 \mathrm{~cm}$, (c) $51 \mathrm{~cm}$, (d) $52.5 \mathrm{~cm}$, (e) $53 \mathrm{~cm}$, and (f) $54 \mathrm{~cm}$.

blue peak generation threshold. When focusing the input beam after the sample we were not able to produce a blueshifted peak [Fig. 4(a)]. For high input energies, the SC extends from the pump wavelength and exhibits a sharp cutoff at $450 \mathrm{~nm}$. By focusing close to the output facet of the sample we observe a peak at $405 \mathrm{~nm}$ [Fig. 4(b)] which may be continuously tuned up to $550 \mathrm{~nm}$ [Figs. 4(b)-4(f)] by simply shifting the sample further away from the focusing lens. We note that exactly the same behavior was reproduced by varying the collimated beam diameter with a variablediameter iris in front of the focusing lens. Decreasing the beam size before the lens leads to increase of the FWHM at the sample input facet and we observe an increase in the blue-peak wavelength.

\section{NUMERICS}

We performed a series of numerical simulations in order to confirm our experimental results and gain a deeper insight into the underlying physics. We used the unidirectional pulse propagation equation solver $[20,21]$ to simulate the pulse evolution and filament creation (see the Appendix for more details). The input pulse was chosen to match the experimentally used pulse albeit with a slightly lower input energy (1 ps pulse at $1.05 \mu \mathrm{m}$ wavelength, collimated to a $28 \mu \mathrm{m}$ beam waist) at the entrance into a fused silica sample and linear chromatic dispersion was modeled by a Sellmeier formula. The $z$ coordinate in all the shown numerical results is referred to the distance within the Kerr sample so that $z=0$ is at the input facet. The nonlinear index value used in these simulations was $2.7 \times 10^{-20} \mathrm{~m}^{2} / \mathrm{W}$, including an $18 \%$ fraction of the stimulated Raman response approximated by a single oscillator with the angular eigenfrequency of 8.3 $\times 10^{13} \mathrm{~s}^{-1}$ and a decay time of $32 \mathrm{fs}$. Multiphoton ionization was described by a simple power-law rate $\approx 10^{-130}\left(\mathrm{~m}^{2} / \mathrm{W}\right)^{8} I^{8}$. Our numerical simulations corroborate that the pulse splitting velocity may be controlled by various modifications of the pump pulse: (i) increasing the pump wavelength or pulse duration in general leads to faster splitting velocities, (ii) use of a flat top or super-Gaussian pulse is 


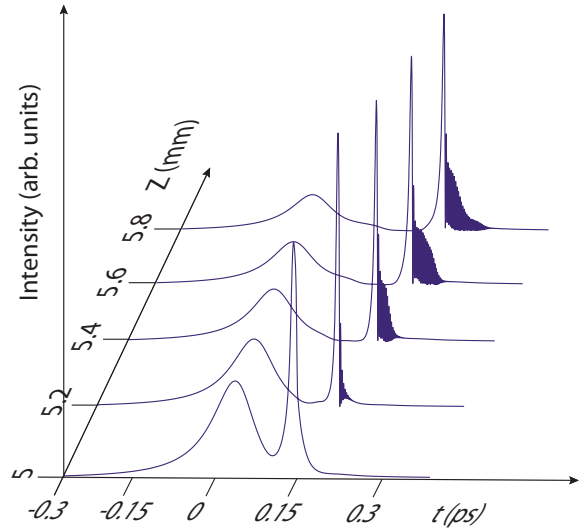

FIG. 5. (Color online) Numerically simulated pulse evolution just after the nonlinear focus. Pulse splitting has occurred and the trailing pulse, for $z=5 \mathrm{~mm}$, is forming a trailing shock front. Further propagation leads to an enhancement of the wave breaking, i.e., the formation of rapid oscillation on the trailing tail.

also very effective in increasing the splitting velocity, (iii) a third possibility is to vary the input beam diameter. Although all three approaches are effective in tuning $v_{X}$ here we focus our attention only on the third strategy that was also adopted in the experiments.

In Fig. 5 we show the numerical evolution of the input pump pulse on-axis intensity profile with an input energy of $5.2 \mu \mathrm{m}$ and a diameter of $5 \mathrm{~mm}$ before the $f=52 \mathrm{~cm}$ focusing lens. Just after the nonlinear focus the pulse splits and starts to form a shock front on the trailing edge of the slower, subluminal pulse. Further propagation leads to marked wave breaking, i.e., oscillations on the tail that, as will be shown further on, are due to the interference between the split daughter pulse and the blue-peak. In Fig. 6 we show the far-field spectra for the same simulation at distances $z$ $=5 \mathrm{~mm}$ and $z=5.8 \mathrm{~mm}$. As may be seen, simultaneously to the shock-front formation the blue peak also appears in the spectrum [Fig. 6(a)] and, as noted elsewhere [16,18], the two

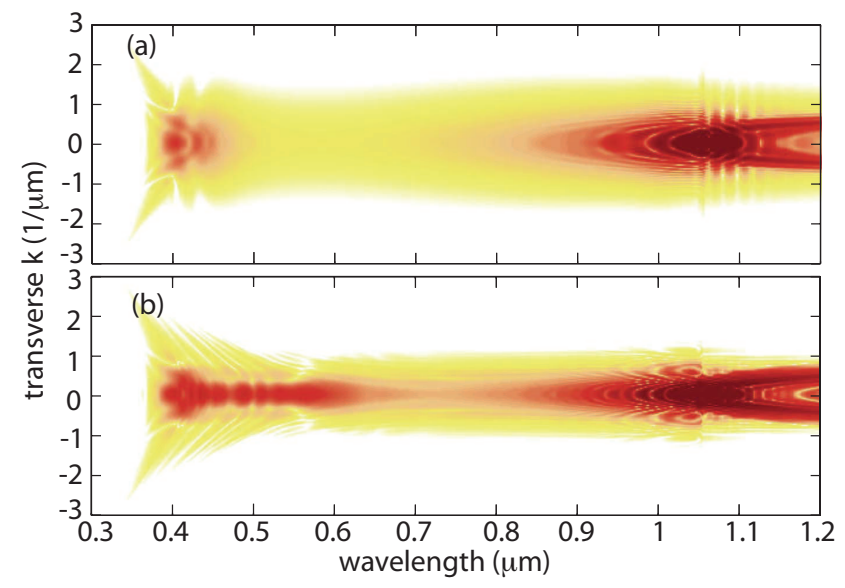

FIG. 6. (Color online) Numerically simulated far-field spectra with an input energy of $5.2 \mu \mathrm{J}$ and an input diameter of $5 \mathrm{~mm}$ before the $f=52 \mathrm{~cm}$ focusing lens and at propagation distances of (a) $5 \mathrm{~mm}$ and (b) $5.8 \mathrm{~mm}$. Both figures are plotted over 4 decades in logarithmic scale.

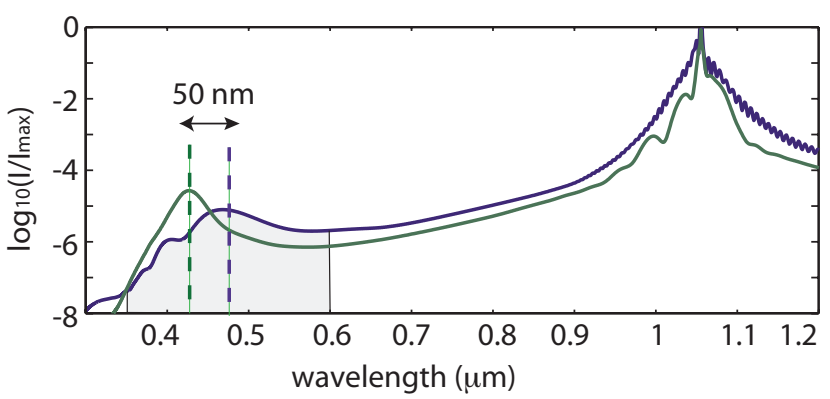

FIG. 7. (Color online) Numerically calculated on-axis spectra for two different input beam diameters: $5 \mathrm{~mm}$ green solid line and $3.3 \mathrm{~mm}$ blue dashed line. The vertical lines indicate the $50 \mathrm{~nm}$ wavelength shift of the blue peak.

are tightly connected. Further propagation leads to broadening of the spectrum centered around the blue-peak wavelength [Fig. 6(b)]. We note the good agreement between the numerically simulated spectra and the experimental spectrum in Fig. 2 confirming that indeed our numerics are capturing the correct physical processes. The first test we then performed was to verify the tunability of the blue-peak wavelength by simply changing the beam diameter before the focusing lens.

Indeed, as seen also in the experiments, such a variation of the input beam leads to a $50 \mathrm{~nm}$ shift of the blue-peak wavelength which is also associated with a corresponding variation in the initial splitting velocity of the input pulse, $v_{X}=2.0273 \times 10^{8} \mathrm{~m} / \mathrm{s}$ and $v_{X}=2.0333 \times 10^{8} \mathrm{~m} / \mathrm{s}$, respectively. We investigated in more depth the link between the blue-peak wavelength and the slow split-off pulse group velocity by directly visualizing the temporal profile evolution of the spectral region close to the blue peak. This was performed by selecting the shaded spectral region in Fig. 7 and by then taking the Fourier transform of the corresponding complex spectral amplitude. By performing this operation on the spectra at various propagation distances it is possible to follow the evolution of the on-axis temporal profile of the blue peak. The results are shown in Fig. 8 for various $z$ and the dashed line shows the position of the trailing pump pulse peak maximum intensity (see also Fig. 5 for the pump pulse temporal profiles). Just after the shock front has formed, at $z=5.2 \mathrm{~mm}$, a sharp, few-cycle pulse appears. This very short pulse arises from the coherent contribution of the continuum of frequencies generated by the steep shock front. As propagation ensues a second, broader, peak appears. By applying a narrow-band filter to the spectra it is possible to determine that this broader peak is associated with the blue peak in the spectrum. Notably, we followed the spectral peak position corresponding to the broad temporal peak and found that, for increasing propagation distance, this peak shifts continuously toward longer wavelengths. This is shown by the green dashed line in Fig. 9. We note that the pump trailing split pulse also exhibits a strong drift in its group velocity as it propagates: the velocity of the pump peak has been explicitly graphed in Fig. 9 as a dotted blue line. As can be seen, the trailing peak velocity is continuously increasing: this may be understood as a drift or relaxation of $v_{X}$ from the strongly subluminal value generated just after the nonlinear focus to- 


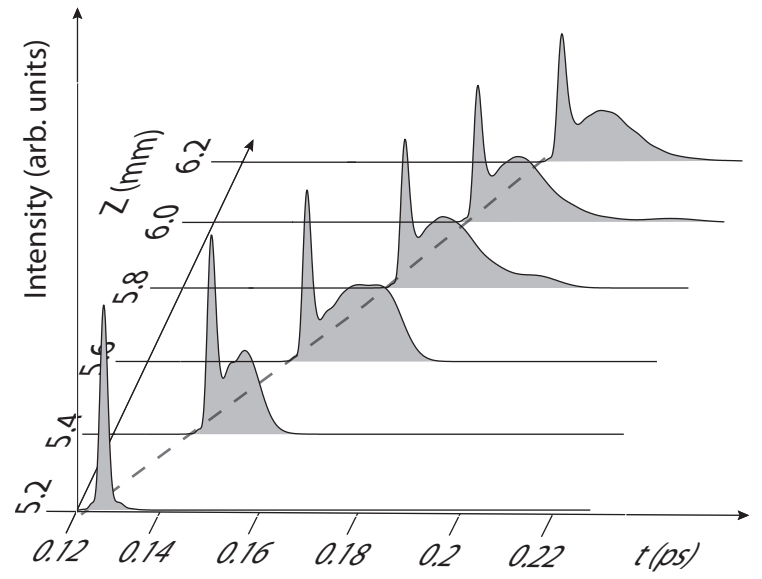

FIG. 8. Evolution of the on-axis temporal profile of the blue peak generated by the pump pulse profiles shown in Fig. 5. The dashed line shows the position of the trailing pump pulse peak maximum intensity.

ward $v_{g}=(d k / d \omega)^{-1}=2.052 \times 10^{8} \mathrm{~m} / \mathrm{s}$. The trailing peak is therefore accelerating and this in turn implies that the phasematching relation (1) predicts a continuously varying wavelength for the blue peak. Using the numerically retrieved values for $v_{X}$ we plot the expected blue peak wavelengths calculated with Eq. (1): the result is the blue solid line in Fig. 9. In the same graph, the green dashed line shows the central wavelength of the blue-peak versus $z$ actually observed in the numerical simulations. As can be seen these two curve are nearly identical thus indicating that the spectral location and evolution of the blue peak is fully determined by the groupvelocity properties and evolution of the trailing pump pulse.

\section{CONCLUSIONS}

In conclusion measurements of ultrashort laser pulse filamentation in bulk fused silica highlight the formation of an

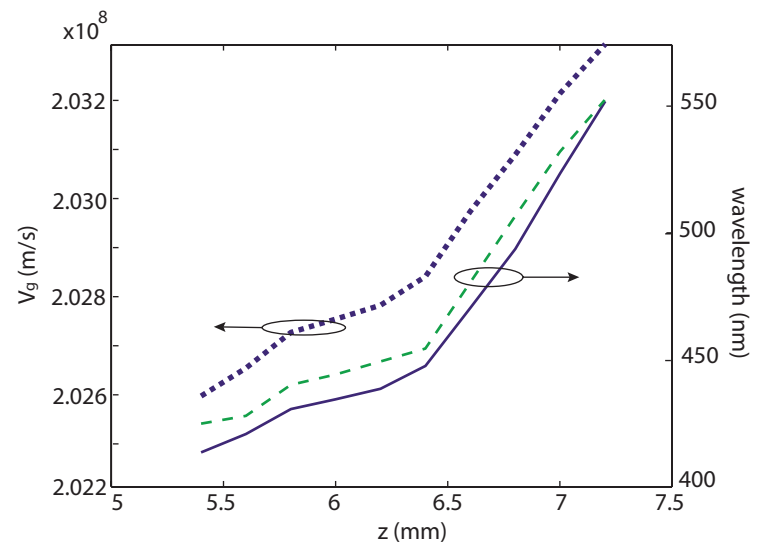

FIG. 9. (Color online) Dashed green line: central wavelength evolution (versus propagation distance $z$ ) of the temporally broad peaks in Fig. 8. Blue dotted line: group velocity evolution of the trailing pump peak. Blue solid line: blue-peak wavelength calculated using the $v_{X}$ values (represented by the dotted line) in the phase-matching relation (1). isolated blue peak. This may be explained by accounting for the particular nature of the $X$ waves associated with the filament. In the light of this understanding, we may fully control SC generation in the blue and uv region of the spectrum: The input-pulse temporal and spatial shaping will affect the filament pulse splitting velocity, or equivalently the $X$-wave velocities, and will in turn modify the SC central wavelength and extent. This will be of fundamental importance for optimizing energy transfer to specific wavelengths and bandwidths according to specific needs, e.g., ultrashort pulse generation, white light spectroscopy or optimized CarrierEnvelope-Offset monitoring in $f$ - $2 f$ setups.

\section{ACKNOWLEDGMENTS}

The authors acknowledge support from the Consorzio Nazionale Interunversitario per le Scienze della Materia (CNISM), project INNESCO. P.D.T acknowledges support from Marie Curie Chair project STELLA, Contract No. MEXC-CT-2005-025710. M.K. and J.V.M. were supported by the Air Force Office for Scientific Research under Grant No. FA9550-07-1-0010.

\section{APPENDIX: PROPAGATION EQUATIONS}

The numerical simulations in this work were performed by solving the unidirectional pulse propagation equations (UPPE) [20,21]. The real electric field for the pulse may be expressed as a plane-wave superposition

$$
E(z, r, t)=\sum_{\vec{k}, \omega} A(z, \vec{k}, \omega) e^{-i \omega t+i \vec{k} \cdot \vec{r}+i K(\omega, k) z},
$$

where the field propagates dominantly along the $z$ axis and $\vec{r}$ is the transverse position vector. The effects of linear medium dispersion on the propagating pulse are fully taken into account through the dispersion relation $K(\omega, k)$ $=\sqrt{\omega^{2} \epsilon(\omega) / c^{2}-k^{2}}$, which gives the $z$ component of the wave vector for an angular frequency $\omega$, and transverse wave vector $k$, for a plane wave propagating in a medium of linear permittivity $\epsilon(\omega)$. The spectral amplitudes $A(z, k, \omega)$ of the field evolve as functions of the propagation distance $z$ solely due to the medium nonlinearities, and obey the UPPE equation

$$
\begin{aligned}
\frac{\partial A(z, k, \omega)}{\partial z}= & +\frac{i \omega^{2} \mu_{0}}{2 K(\omega, k)} \int e^{i[\omega t-\vec{k} \cdot \vec{r}-K(\omega, k) z]} P(z, r, t) d^{2} r d t \\
& -\frac{\omega \mu_{0}}{2 K(\omega, k)} \int e^{i[\omega t-\vec{k} \cdot \vec{r}-K(\omega, k) z]} J(z, r, t) d^{2} r d t
\end{aligned}
$$

The nonlinear polarization $P=\epsilon_{0} \Delta \chi E$ depends on the propagating optical field through the medium nonlinearity, where $\Delta \chi(z, r, t)$ is the local modification of the medium's optical susceptibility caused by a combined action of the Kerr and stimulated Raman effects 


$$
\Delta \chi=2 n_{b} n_{2}\left[(1-f) E^{2}+f \int_{0}^{\infty} \mathcal{R}(\tau) E^{2}(t-\tau) d \tau\right] .
$$

Here, $\mathcal{R} \sim \sin (\Omega \tau) e^{-\Gamma \tau}$ is the memory function of the stimulated Raman effect.

The effects due to the free electrons generated by ionization in the high intensity optical field are incorporated through the current density $J(z, r, t)$ modeled by Drude model

$$
\partial_{t} J(z, r, t)=-\frac{1}{\tau} J(z, r, t)+\frac{e^{2}}{m_{e}} \rho(z, r, t) E(z, r, t),
$$

where the free electron density $\rho(z, r, t)$ is generated by multiphoton ionization, while electron recombination and diffusion is neglected on the femtosecond time scales. Our current density also includes a phenomenological component that mimics the multiphoton ionization losses. Parameters of the medium model were as in Ref. [19].
[1] J. Kasparian, M. Rodriguez, G. Mèjean, J. Yu, E. Salmon, H. Wille, R. Bourayou, S. Frey, Y.-B. Andrè, A. Mysyrowicz, R. Sauerbrey, J. P. Wolf, and L. Wöste, Science 301, 61 (2003).

[2] C. P. Hauri, W. Kornelis, F. W. Helbing, A. Heinrich, A. Couairon, A. Mysyrowicz, J. Biegert, and U. Keller, Appl. Phys. B 79, 673 (2004).

[3] R. R. Alfano, The Supercontinuum Laser Source (SpringerVerlag, New York, 1989).

[4] K. D. Moll, A. L. Gaeta, and G. Fibich, Phys. Rev. Lett. 90, 203902 (2004).

[5] M. A. Porras, A. Parola, D. Faccio, A. Couairon, and P. Di Trapani, Phys. Rev. A 76, 011803(R) (2007).

[6] A. Couairon and A. Mysyrowicz, Phys. Rep. 441, 47 (2007).

[7] S. Trillo, C. Conti, P. Di Trapani, O. Jedrkiewicz, J. Trull, G. Valiulis, and G. Bellanca, Opt. Lett. 27, 1451 (2002).

[8] D. Faccio, M. A. Porras, A. Dubietis, F. Bragheri, A. Couairon, and P. Di Trapani, Phys. Rev. Lett. 96, 193901 (2006).

[9] D. Faccio, P. Di Trapani, S. Minardi, A. Bramati, F. Bragheri, C. Liberale, V. Degiorgio, A. Dubietis, and A. Matijosius, J. Opt. Soc. Am. B 22, 862 (2005).

[10] M. A. Porras and P. Di Trapani, Phys. Rev. E 69, 066606 (2004).

[11] P. Di Trapani, G. Valiulis, A. Piskarskas, O. Jedrkiewicz, J.
Trull, C. Conti, and S. Trillo, Phys. Rev. Lett. 91, 093904 (2003).

[12] M. Kolesik, E. M. Wright, and J. V. Moloney, Phys. Rev. Lett. 92, 253901 (2004).

[13] A. V. Yulin, D. V. Skryabin, and P. St. J. Russell, Opt. Lett. 30, 525 (2005).

[14] F. Bragheri, D. Faccio, A. Couairon, A. Matijosius, G. Tamosauskas, A. Varanavicius, V. Degiorgio, A. Piskarskas, and P. Di Trapani, Phys. Rev. A 76, 025801 (2007).

[15] D. Faccio, A. Averchi, A. Couairon, A. Dubietis, R. Piskarskas, A. Matijosius, F. Bragheri, M. A. Porras, A. Piskarskas, and P. Di Trapani, Phys. Rev. E 74, 047603 (2006).

[16] J. Liu, R. Li, and Z. Xu, Phys. Rev. A 74, 043801 (2006).

[17] M. Kolesik, E. M. Wright, and J. V. Moloney, Opt. Express 13, 10729 (2005).

[18] V. Kartazaev and R. R. Alfano, Opt. Lett. 32, 3293 (2007).

[19] D. Faccio, A. Averchi, A. Couairon, M. Kolesik, J. V. Moloney, A. Dubietis, G. Tamosauskas, P. Polesana, A. Piskarskas, and P. Di Trapani, Opt. Express 15, 13077 (2007).

[20] M. Kolesik, J. V. Moloney, and M. Mlejnek, Phys. Rev. Lett. 89, 283902 (2002).

[21] M. Kolesik and J. V. Moloney, Phys. Rev. E 70, 036604 (2004). 